\title{
PARTICLE SIZE DISTRIBUTION CORRECTION METHOD USING A SIMULATED ANNEALING TECHNIQUE
}

\author{
A. N. Diógenes ${ }^{a}$, \\ L. O. E. dos Santos, \\ and C. P. Fernandes ${ }^{b}$ \\ ${ }^{a}$ Universidade Federal do Paraná, \\ Departamento de Engenharia Mecânica, \\ Bairro Jardim das Américas \\ CP. 19011, Curitiba, Paraná, Brasil \\ andiogenes@ufpr.br \\ ${ }^{\mathrm{b}}$ Universidade Federal de Santa Catarina, \\ Laboratório de Meios Porosos e Propriedades \\ Termofísicas - LMPT \\ CEP 88040-900 - C.P. 476 \\ Florianópolis, SC, Brasil
}

\section{NOMENCLATURE}

d particle diameter

E simulated annealing Energy

f diameter frequency

$\mathrm{n}$ number of spheres

$\mathrm{p} \quad$ simulated annealing probability

$\mathrm{r} \quad$ 2D circle radius

$\mathrm{R} \quad 3 \mathrm{D}$ sphere radius

$\mathrm{T}$ simulated annealing temperature

$\mathrm{x} \quad$ function adjustment coefficient 1

\section{Greek symbols}

function adjustment coefficient 2

$\beta \quad$ function adjustment coefficient 3

$\delta \quad$ function adjustment coefficient 4

$\mu \quad$ function adjustment coefficient 5

$\eta \quad$ function adjustment coefficient 6

$\sigma \quad$ function adjustment coefficient 7

\section{Subscripts}

$\mathrm{t} \quad$ iteration counter

\section{INTRODUCTION}

\section{ABSTRACT}

The procedure for obtaining the particle size distribution by visual inspection of a sample involves stereological errors, given the cut of the sample. A cut particle, supposedly spherical, with radius $R$, will be counted as a circular particle with radius $r, r \leq R$. The difference between $r$ and $R$ depends on how far from the center of the sphere the cut was performed. This introduces errors when the extrapolation of the properties from two to three dimensions during the analysis of a sample. The usual method is to correct the distribution by probabilistic functions, which have large errors. This paper presents a method to reduce the error inherent to this problem. The method is to compute a simulation of the preparation process in a sample whose structure can be described by non-penetrating spheres of various diameters which meet a known probability distribution function, for example, a log-logistic function, or even a constant function. For each distribution radius, a number of spheres is generated and virtually cut, generating a bi-dimensional (2D) distribution. The 2D curves of the spheres distribution obtained in this simulation are compared with that obtained by the experimental procedure and then the parameters of the threedimensional distribution function are adjusted until the 2D curves are similar to the experimental one using the optimization method Simulated Annealing for the curve-fitting. In future this method will be applied to the analysis of the oil reservoir rocks.

Keywords: Simulated annealing, Porous media, Curve-fitting, Stereology
The stereology is a interdisciplinar study field which aims to evaluate the three dimensional (3D) properties of a sample using a material bidimensional (2D) information. Several geometrics and statistical methods are applied to achieve this target (DeHoff and Rhines, 1968).

The stereology has many applications in biology, medical sciences, material sciences, and wherever it is necessary to obtain information of dimensions higher from samples with inferior dimensions. The stereology challenge is to understand the structural inner three-dimensional arrangement based on the analysis of the structure slices that show only two-dimensional information for that stereological principles take into consideration geometry and probability statistics (Mandarin-De-Lacerda, 2003).

It is inherent, however, to the stereological technique, the introduction of errors during the extrapolation process. Garcia et. al. (2007) state that A major problem of sampling/slicing is the loss of dimension; a 3D object becomes a 2D area, a surface (2D) becomes a line (1D), a line (1D) becomes a point (OD) and a number (points 0D) is lost. A stereological approach provides solutions to this.

In the last 50 years, stereological studies have appeared in literature more and more frequently, the first studies being based on pioneers (Chalkley 1943, Abercrombie 1946, Chalkley et al. 1949, Weibel and Gomez 1962, Weibel et al. 1966). The so called "new 
stereology" was developed in the XXth century's eighties, a collection of procedures turning stereology easier and more unbiased (Gundersen et al. 1983, Sterio 1984, Gundersen 1988) - in reality, the question of bias and stereology is still under discussion, but new techniques make stereology more consistent (Hedreen 1999, Baddeley 2001, von Bartheld 2002, Gardella et al. 2003, Mandarin-DeLacerda, 2003).

On the other hand, the idea of estimating stereological parameters from optical sections within a thick slice was first used for counting particles in optical disector principle (Sterio, 1984) and in unbiased sampling brick rule (Howard et al., 1985), and then in many other stereological methods, e.g., nucleator (Gundersen, 1988) and planar rotator (Jensen and Gundersen, 1993) applied to a stack of optical sections and estimating the mean particle volume, spatial grid (Sandau, 1987), methods for estimating the surface area, method of vertical slices (Gokhale, 1990), and global spatial sampling (Larsen et al., 1998) used for the length estimation.

Indeed, stereology is dynamic and full of perspectives for the future, new approaches to old questions still stimulates stereologists to test possibilities, an exciting example is the Simulated annealing technique for curve fitting.

This research evaluated several functions as candidates to represent 3D particle size distributions which generated 2D particle size distributions experimentally measured through rock samples cut and image analysis.

The evaluation determined the most adequate function for representing the particle size distribution and a new alternative was also proposed: not using a specific function, but to optimize a constant distribution.

A comparison between the classical SchwartzSaltykov (DeHoff and Rhines, 1968), the

\section{METHODOLOGY}

\section{Experimental particle size distribution}

The experimental procedure to obtain a particle size distribution was the same as Diógenes et. al. (2009). A typical particle diameter distribution can be observed in Figure (1).

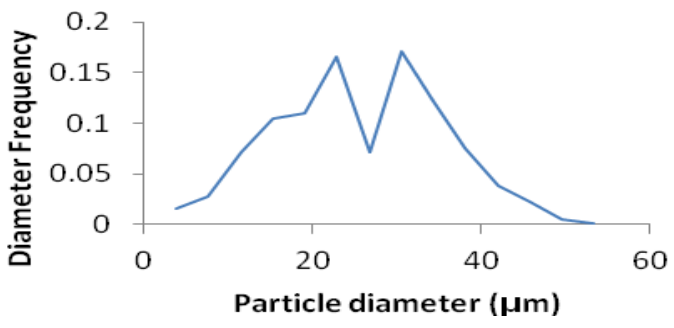

Figure 1. Typical particle size distribution for a sandstone sample obtained by image analysis.

\section{Particle size distribution modeling}

To model a particle size distribution, it was done some considerations:

- each particle is represented by a sphere;

- the spheres are non-penetrating;

- the 3D particle size distribution follows a known unimodal distribution, as a log-logistic function with unknown parameters which shall be optimized by a Simulated Annealing method;

These considerations aim to make the sample particles model as real as possible, but yet feasible to optimize. The unimodal function consideration intends to simplify the optimization procedure, even if the Figure (1) can be considered a bimodal function, it is considered that the oscillation around the $30 \mu \mathrm{m}$ diameter is a fluctuation.

The functions chosen to model the particle size distribution were the following:

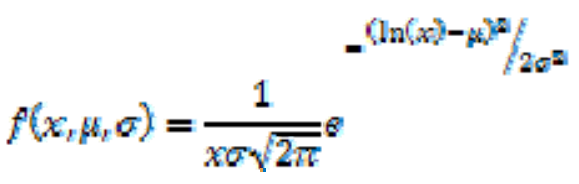

$f(x, \beta, \beta)=\frac{\sigma^{\left(-\frac{\alpha-\mu}{\beta}\right)} \sigma^{-\left(-\frac{\alpha-\alpha}{\beta}\right)}}{\beta}$

$$
\begin{aligned}
& f(x, \beta, n, \delta)=\beta e^{-\beta(x+\delta)} e^{-n e^{-\beta(x+\delta)}}\{1 \\
& \left.+n\left[-e^{-\beta(x+\delta)}\right]\right\}
\end{aligned}
$$

$$
f\left(x_{\varepsilon}, \varepsilon_{z} \beta\right)=\frac{\left(\frac{\beta}{\alpha}\right)\left(\frac{x}{\sigma}\right)^{\beta-1}}{\left[1+\left(\frac{x}{\alpha}\right)^{\beta}\right]^{2}}
$$

These functions, Log-Normal - Eq. (1), FischerTippet - Eq. (2), Shifted-Gompertz - Eq. (3) and Log-Logistic Eq. (4), were chosen as unimodal functions and due to its flexibility. The log-normal function, for example in Figure (2) for different values of $\sigma$, can have several shapes, depending on the variables. 


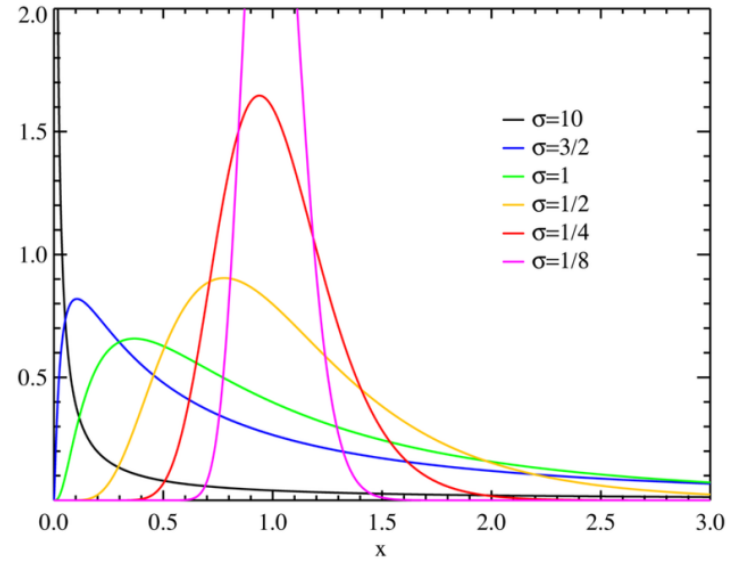

Figure 2. Log-Normal function shapes varying the $\sigma$ parameter.

For all these functions the function parameters were randomized and a 3D initial particle size distribution was generated.

The 2D particle size distribution was generated through virtual sphere slices. For each distribution radius, a number of $10^{5}$ times the distribution spheres was generated. These spheres are then cut and 2D particles (circles) with smaller radius are then created, as shown in Figure (3). $\mathrm{R}$ is the real radius, $\mathrm{r}$, the measured radius and $h$ is the distance from the center where the slicing plane cut the particle. For example, a particle size distribution which has 0.01 $(1 \%)$ of its particles of radius 3 , will have, for this radius, 1.000 spheres generated, which will be virtually cut and these cuts can create $2 \mathrm{D}$ particles measurements with radius 3,2 and 1 .

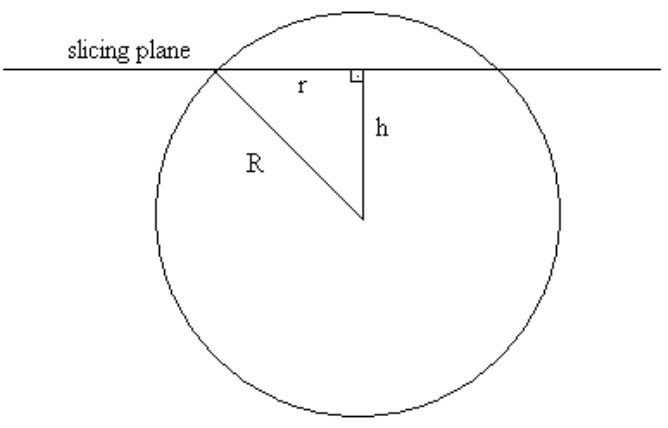

Figure 3. Sphere (Particle) cut by a slicing plane illustrating the measurement of a $r$ radius, smaller than R.

Both the 2D particle size distribution created by a $3 \mathrm{D}$ particle size distribution can be observed in the Figure (4) for a Log-Normal distribution. As expected, the created $2 \mathrm{D}$ curve has a higher frequency of particles with smaller diameter than the 3D curve.

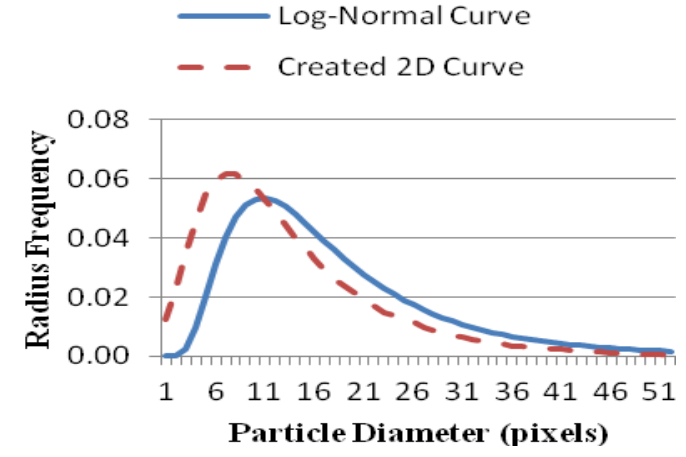

Figure 4. Log-normal curve and the created 2D after the virtual cuts.

\section{Simulated Annealing (SA) Algorithm}

The well-known SA technique (Yeong and Torquato 1998a,b; Manwart et al. 2000; Talukdar and Torsaeter 2002; Talukdar et al. 2002a,b; Hamzehpour and Sahimi 2006, Arpat and Caers 2007, Capek et al. 2008) was used to optimize the $3 \mathrm{D}$ particle size distribution.

Once the $3 \mathrm{D}$ particle size distribution is generated, a SA algorithm is used to adjust the curve parameters to fit the $2 \mathrm{D}$ gran size distribution. The sequence of steps to optimize the curve is described below:

1. generate a $3 \mathrm{D}$ particle size distribution with random parameters;

2. execute a virtual slicing procedure, generating a 2D particle size distribution;

3. compare the experimental and generated 2D particle size distributions, calculating a square error;

4. modify the $3 \mathrm{D}$ particle size distribution parameters and accept or reject the modification according to the SA algorithm;

5. go to step 2 until convergence or iteration limit is reached.

Notice that the optimization is carried on two levels. While the 3D curve is directly modified, the $2 \mathrm{D}$ generated curve provides the square error. This configuration is due the experimental procedure, which provides a $2 \mathrm{D}$ particle size distribution, so it is necessary to compare both $2 \mathrm{D}$ distributions. The square error was calculated as the following equation:

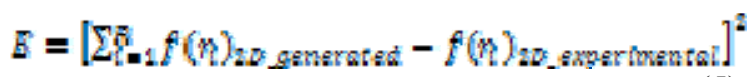

The SA algorithm used in this research is the classical algorithm with linear cooling schedule, 
having $T_{\tau+1}=0.99 T_{\tau}$. The cooling happened after 1000 well succeeded iterations.

The parameter modification was executed at one parameter per iteration. For the Log-Normal function, for example, if the $t$ iteration modified the $\mu$ parameter, the $t+1$ iteration modified the $\sigma$ parameter. The modification step was 0.01 and it could be added or subtracted with $50 \%$ of chance for each option. The convergence target was $10^{-3}$ for the square error. The iteration limit was 10.000 iterations without improvement in the energy function (square error).

The technique cost less than one minute to optimize a curve using an Intel ${ }^{\circledR}$ i3 $2.933 \mathrm{GHz}$ processor.

\section{RESULTS AND DISCUSSION}

A typical result for the same particle size distribution from Figure (1) can be observed in Figure (5). The square error for this result was $9 \times 10^{-4}$.

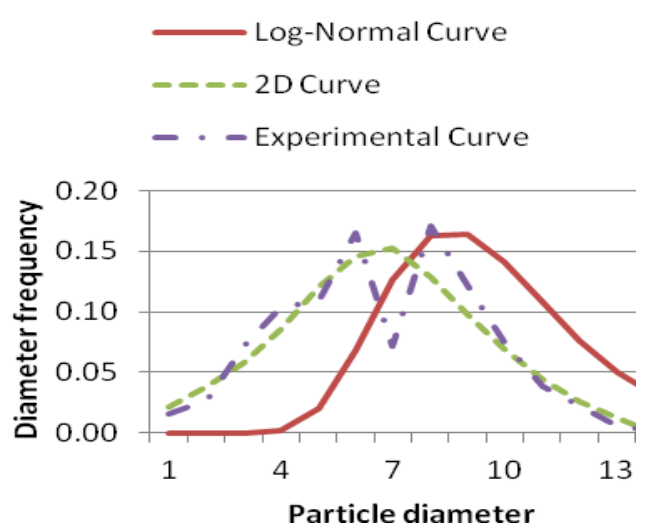

Figure 5. Processing result for the particle size distribution exposed in Figure (1).

To test which function could model better the particle size distribution, a SA process was executed for 21 particle size distributions for all the adopted functions without ending the processing with the convergence criteria. An average square error for all the samples can be observed in the Table (1). The function with the best result was the Log-Logistic. The obtained square error was 7.63E-04. This indicates that the best function for representing the particle size distribution among the analyzed functions is the Log-Logistic.

Table 1. Average square error for all the 21 rock samples processed without convergence criteria stop.

\begin{tabular}{c|c}
\hline Function & $\begin{array}{c}\text { Average square error } \\
\text { obtained }\end{array}$ \\
\cline { 1 - 1 } Log Normal & $1.03 \mathrm{E}-03$ \\
\hline Shifted Gompertz & $8.56 \mathrm{E}-04$ \\
\cline { 1 - 1 } Fischer Tippet & $8.14 \mathrm{E}-04$ \\
\cline { 1 - 1 } Log Logistic & $7.63 \mathrm{E}-04$
\end{tabular}

To compare the proposed methodology with classic methods, a Log-Normal distribution with 10 particle diameters, $\mu=1.3$ and $\sigma=0.4$ was adopted as a $3 \mathrm{D}$ particle size distribution and the virtual cut was executed. The resultant distributions are shown in Figure (6).

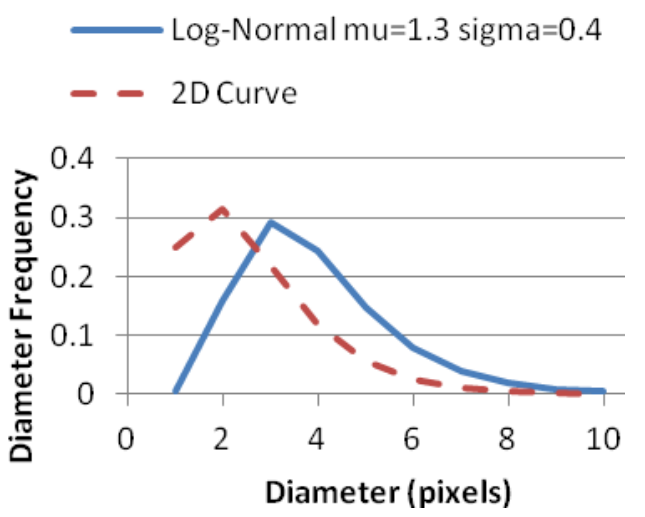

Figure 6. Log-Normal curve with 10 particle diameters, $\mu=1.3$ and $\sigma=0.4$ and $2 \mathrm{D}$ curve resultant from virtual cuts.

The classical method of Schwarz-Saltykov (DeHoff and Rhines, 1968), which could be adapted for a curve correction, was applied to the resultant 2D curve and a 3D corrected particle size distribution was generated. The latter was compared with a particle size distribution generated using the proposed methodology through quadratic error calculated in the 3D distribution. The corrected distributions and the resulting curves are disposed in the Figure (7). The quadratic error for the Schwarz-Saltikov method was $2.2 \mathrm{E}-1$, while the function $\mathrm{SA}$ was $8 \mathrm{E}-2$, approximately 5 times smaller error.

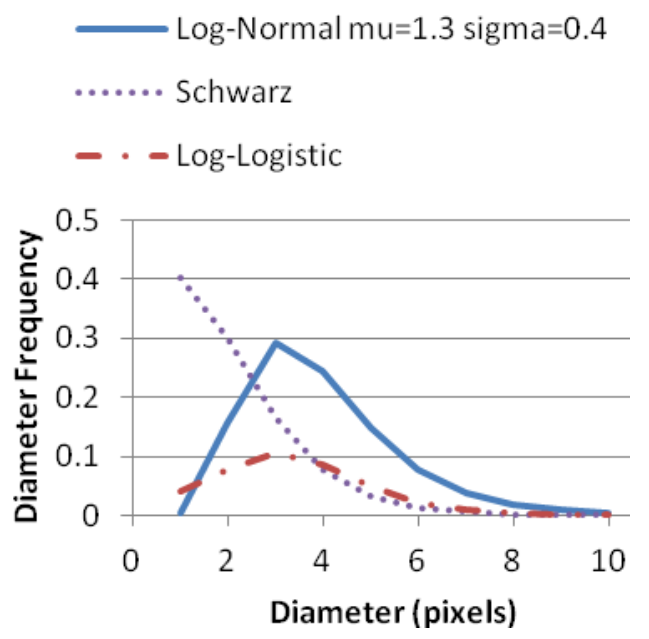

Figure 7. Comparison between 3D particle size distributions generated by the proposed methodology and the classical Schwarz-Saltikov method. 
Notice that even if the error was almost 5 times smaller, the 3D distribution was not satisfactory. The function SA was not able to represent a Log-Normal curve with a Log-Logistic curve, even if both distributions are theoretically compatible.

A proposed solution is to not use a function to represent the $3 \mathrm{D}$ particle size distribution. Instead, it can be used a constant function or a linear function and let the SA algorithm modify it freely. A preliminary result of a constant function modifying with modifying step 1E-3 is shown in Figure (8). The $2 \mathrm{D}$ input was the same 2D Log-Normal as the previous example and the $3 \mathrm{D}$ result is compared with the previous Log-Normal.

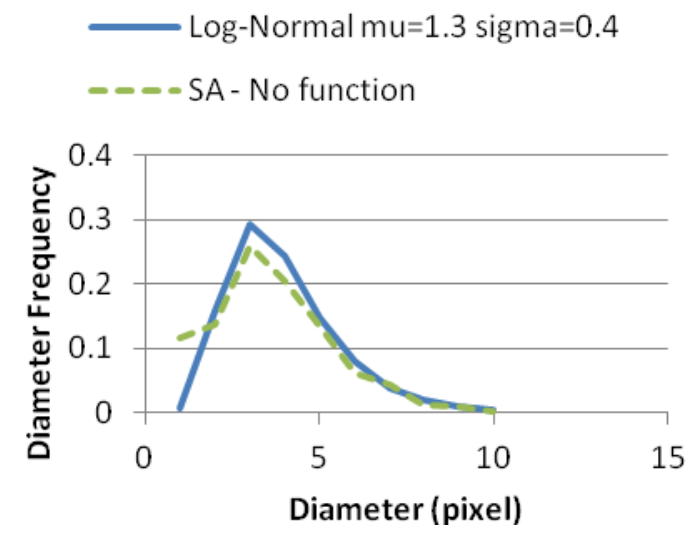

Figure 8. Comparison between 3D particle size distribution: generated by the SA-no function and previous Log-Normal function.

There is a significant agreement between both functions. The quadratic error for this methodology was $1 \mathrm{E}-2$, even smaller than the SA with a function representation. This methodology was also tested with the gran size distribution showed in Figure (1). The resulting curves are exposed in Figure (9).

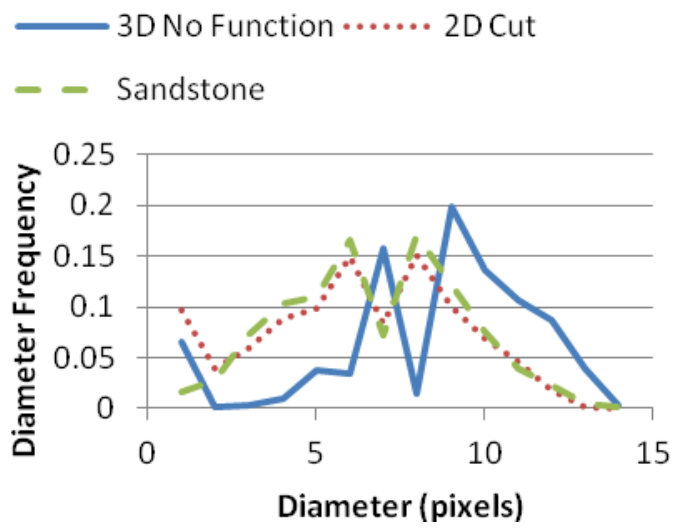

Figure 9. 3D Curve generated using a SA technique without optimizing a specific function and its $2 \mathrm{D}$ cut comparing to a sandstone grain size distribution.
An interesting fact is that the new methodology was able to reproduce a bimodal function and the 3D generated curve was also bimodal. This new methodology shall be further investigated.

\section{CONCLUSIONS}

It was proposed a new methodology to model particle size distributions. A SA algorithm was used to fit a 3D distribution with virtual cuts and minimizing a 2D function, comparing this latter with an input grain size distribution obtained experimentally.

It was tested 4 distribution functions, LogNormal, Fischer-Tippet, Shifted-Gompertz and LogLogistic. The function which had best performance was the Log-Logistic distribution, yet, in a simple test of flexibility, it was clear that the performance of the function fitting was not adequate.

A solution was proposed, which is to use a SA algorithm to fit freely a constant function. The preliminary tests showed that this algorithm was able to reproduce both a $3 \mathrm{D}$ Log-Normal function and a bimodal input function, indicating that it can be used to represent 3D particle size distributions determined from $2 \mathrm{D}$ particle size distributions. This solution shall be further investigated in future researches.

\section{ACKNOWLEDGEMENTS}

The authors acknowledge the Agência Nacional do Petróleo - ANP for funding this this research and the Petrobras Research Facility (CENPES) for providing the microtomographies used in this research.

\section{REFERENCES}

Abercrombie M. 1946. Estimation of nuclear population from microtomic sections. Anatomical Record, Vol. 94, No. 2, pp. 239-247

Arpat, G.B. and Caers, J., 2007, Conditional simulation with Patterns, Mathematical Geology, Vol. 39, No. 2, pp. 177-203

Baddeley A. J. 2001. Is stereology 'unbiased'? Trends in Neuroscience, Vol. 24, No. 7, pp. 375-376, author reply pp. 378-380

Čapek, P. Hejtmánek, V. Brabec, L. Zikánová A. and Kočiřík M., 2008, Stochastic Reconstruction of Particulate Media Using Simulated Annealing: Improving Pore Connectivity, Transport in Porous Media, Vol. 76, No. 2, pp. 179-198

Chalkley H. W. 1943. Methods for quantitative morphological analysis of tissue. Journal of Natural Cancer Institute, Vol. 4, No. 1, pp. 47-53.

Chalkley H. W., Cornfield J. and Park H. C. 1949. A method for estimating volume-surface ratios. Science, Vol. 110, pp. 295-301

DeHoff, R. T.; Rhines, F. N., 1968, Quantitative Microscopy. McGraw-Hill Book Company. 
Diógenes, A. N., dos Santos, L. O. E., Fernandes, C. P., 2009, Porous media microstructure reconstruction using pixel-based and object-based simulated annealing - comparison with other reconstruction methods. Reterm Engenharia Térmica, Vol. 8, No. 2, pp. 35-41

Garcia, Y., Breen, A., Burugapalli, K., Dockery, P., Pandit, A. 2007. Stereological methods to assess tissue response for tissue-engineered scaffolds. Biomaterials, Vol. 28, No. 2, pp. 175-186.

Gardella D., Hatton W. J., Rind H. B., Glenn G. D. and Von Bartheld C. S., 2003. Differential tissue shrinkage and compression in the z-axis: implications for optical disector counting in vibratome-, plasticand cryosections. Journal of Neuroscience Methods Vol. 124, No. 1, pp. 45-59

Gardi, J.E., Nyengard, J. R., Gundersen, H.J.G., 2006. Using unbiased image analysis for improving unbiased stereological number estimates - a pilot simulation study using the smooth fractionator, Journal of Microscopy, 2006, Vol. 222, Pt. 3, pp. 242-250

Gokhale, A.M. 1990. Unbiased estimation of curve length in $3 \mathrm{D}$ using vertical slices. Journal of Microscopy. Vol. 159, No. 2, pp. 133-141.

Gundersen H. J. 1988. The nucleator. Journal of Microscopy, Vol. 151 (Pt 1), pp. 3-21

Gundersen H. J., Andersen B. S. and Floe H. 1983. Estimation of section thickness unbiased by cutting-deformation. Journal of Microscopy, Vol. 131 (Pt 1), pp. 3-4

Hamzehpour, H., Sahimi, M., 2006, Development of optimal models of porous media by combining static and dynamic data: The porosity distribution. Physical Review. E 74, 026308

Hedreen J. C. 1999. Unbiased stereology? Trends in Neuroscience, Vol. 22, No. 8, pp. 346-347

Howard, C.V., Reid, S., Baddeley, A., Boyde, A. 1985. Unbiased estimation of particle density in the tandem scanning reflected light microscope. Journal of Microscopy, Vol. 138, No. 2, pp. 203-212.

Jensen, E.B.V., and Gundersen, H.J.G. 1993. The rotator. Journal of Microscopy. Vol. 170, No. 1, pp. 35-44.

Larsen, J.O., Gundersen, H.J.G., and Nielsen, J. 1998. Global spatial sampling with isotropic virtual planes: estimators of length density and total length in thick, arbitrarily orientated sections. Journal of Microscopy. Vol 191, No. 3, pp. 238-248.

Mandarim-De-Lacerda, C. A. 2003. Stereological tools in biomedical research, in: Anais da Academia Brasileira de Ciências, Rio de Janeiro, Vol. 75, No. 4

Manwart, C., Torquato, S., Hilfer, R., 2000, Stochastic reconstruction of sandstones, Physical Review E, Vol. 62, No 41Sterio D. C. 1984. The unbiased estimation of number and sizes of arbitrary particles using the disector. Journal of Microscopy, Vol. 134 (Pt 2), pp. 127-136
Sandau, K. 1987. How to estimate the area of a surface using a spatial grid. Acta Stereologica. Vol. 6. pp. 31-36.

Talukdar, M.S., Torsaeter, O., 2002, Reconstruction of chalk pore networks from 2D backscatter electron micrographs using a simulated annealing technique, Journal of Petroleum Science Engineering, Vol. 33, No. 2, pp. 265-282

Talukdar, M.S., Torsaeter, O., Ioannidis, M.A., Howard, J.J. 2002a, Stochastic reconstruction, 3D characterization and network modeling of chalk. Journal of Petroleum Science Engineering, Vol. 35 No 1-2, pp. 1-21

Talukdar, M.S., Torsaeter, O., Ioannidis, M.A., Howard, J.J., 2002b, Stochastic reconstruction of chalk from 2D images, Transport in Porous Media Vol. 48, No. 1, pp. 101-123

Von Bartheld C. 2002. Counting particles in tissue sections: choices of methods and importance of calibration to minimize biases. Histological Histopathology, Vol. 17, No. 2, pp. 639-648

Weibel E. R and Gomez D. M. 1962. A principle for counting tissue structures on random sections. Journal of Applied Physiology, Vol. 17, pp. 343-348

Weibel E. R, Kistler G. S., and Scherle W. F. 1966. Practical stereological methods for morphometric cytology. Journal of Cell Biology, Vol. 30, pp. 23-38.

Yeong, C.L.Y. and Torquato, S., 1998a, Reconstructing random media, Physical Review E Vol. 58, No. 1, pp. 495-506

Yeong, C.L.Y. and Torquato, S., 1998b, Reconstructing random media II - Three-dimensional media from two dimensional cuts, Physical Review E Vol. 58, No. 1, pp. 224-233

\footnotetext{
Received: July 30, 2011

Revised: August 30, 2011

Accepted: September 30, 201
} 